\title{
CAS Highlighted in Annual Top 10 Science Advances of China for 2019
}

\author{
By SONG Jianlan and YAN Fusheng (Staff Reporters)
}

$\mathrm{O}$ n February 27, 2020, the Chinese Ministry of Science and Technology announced the widely awaited annual list of the top 10 science advances of China for the past year. A discovery made by Chang'e-t, the lunar mission that made the first successful soft landing on the far side of the Moon, ranks first on the rigorously competitive list. Among the winners are also some other high-profile projects. including the identification of a fossilized mandible of Denisovan from the Qinghai-Tibet Plateau, which was listed in the annual breakthroughs of 2019 by Science.

The election reviewed the science discoveries published during the period from December 1, 2018 to November 30, 2019, and chose 10 out of 320 candidates from different areas of natural sciences. Over 2.600 leading scientists in China, including Members of the Chinese Academy of Sciences (CAS) and the Chinese
Academy of Engineering, principal investigators of major projects such as the National Basic Research Program (dubbed "97.3 Program”) and directors of State Key Laboratories, had their say in the two rounds of ballots to decide the final winners.

As an effort to encourage science exploration in the country, and to boost science awareness in the public, the Chinese academic community has been celebrating science excellence by recognizing the most important domestic research advances every spring since 2006.

Once again, discoveries made by CAS scientists dominate the list for the year 2019. Seven out of the 10 places, namely the $1^{\text {st }}, 4^{\text {th }}, 5^{\text {th }}, 7^{\text {th }}, 8^{\text {th }}, 9^{\text {th }}$ and the $10^{\text {th }}$ are taken by advances made by CAS scientists as principal investigators or major contributors.

$B C A S$ is introducing the winner projects accomplished by CAS scientists here as follows.

\section{Initial Evidence of Lunar Mantle Composition Detected by Chang'e-4}

Previous research suggested that the lunar crust as well as the mantle could both have formed at the infantile stage of the Moon's evolution. At this stage, major crashes and accretion process generated a great amount of energy that melted down the substance and gave birth to a magma ocean. When this ocean of different minerals cooled down, the lighter substances, represented by the calcium-rich plagioclase, crystallized and rose to shape the crust, while the heavier ones, like the femic minerals including orthopyroxene and olivine, sank down to form the mantle.

Curiously, the lunar samples returned by the Apollo and Luna missions did not give any direct information

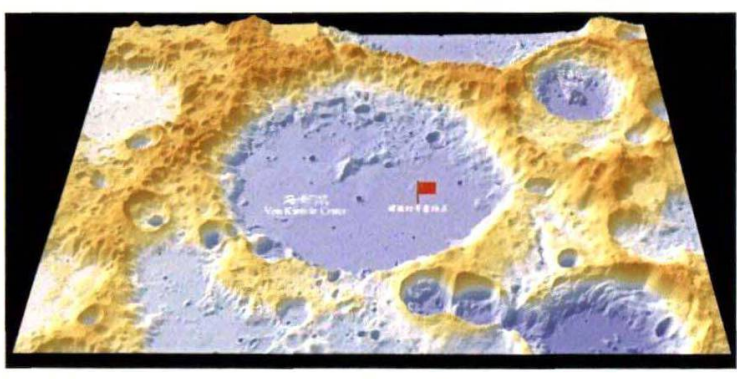

On January 9, 2019, Chang'e-4, a mission under China's Lunar Exploration Program, touched down on the eastern floor of the Von Kármán crater of the Moon's far side, and sent hours later the rover Yutu-2 to perform an in-situ investigation of the local environment, the first ever made by a manmade device. It successfully detected initial evidence for the composition of the lunar mantle. Shown is the landing site with mantle material buildup. (Credit: CLEP/NSSC) 
about the substantial composition of the mantle, leaving the above-mentioned inference unsupported for a long time.

The mission Chang'e-t under China's Lunar Exploration Program (CLEP), helped to fill this gap with its in-situ investigation - the first ever by a manmade spacecraft - in the South Pole-Aitken basin (SPA) on the far side of the Noon, unveiling the first clips of this immersed world. Read more on page 8.

\section{Unlocking the Structure and Function of Light-harvesting Complexes in Algae}

Oxygenic photosynthetic organisms harness sunlight to convert carbon dioxide and water into carbohydrates and molecular oxygen, which are essential to nearly all living things on the planet. And it has been a longlasting quest for scientists to reveal the mystery of how exactly plants and algae harvest and convert light energy into chemical energy and atmospheric oxygen.

To solve this mystery, scientists at the CAS Institute of Botany have made breakthroughs in elucidating many fine structures of the protein complexes that are responsible for light energy harvesting, transfer, and dissipation. These structures provide a solid basis for revealing the mechanisms of how lights are harvested and converted in

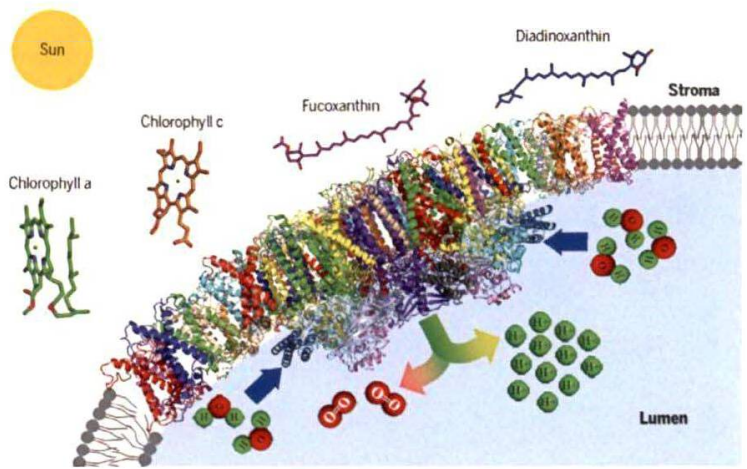

Model of a diatom pigment-protein supercomplex embedded in the thylakoid membrane where photosynthetic reactions occur. (Credit: Science) nature. For more details, please refer to page 10.

\section{$5^{\text {th }}$}

\section{Combinatorial Chemistry Yields High-temperature Bulk Metallic Glasses}

Metallic glasses also known as amorphous or glassy alloys, are considered to be the next-generation materials following steel and plastic. They are strong, shapeable as plastic and corrosion resistant; like metals, they can also conduct heat and electricity. All the properties make them ideal for fashioning gears. precision molds and electrochemical devices, as well as key parts in many high-tech fields such as aerospace and national defense.

Though the first metallic glass was made in 1960. there are only a dozen of metallic glasses so far showing practical applicability. Because the discovery of metallic glasses with specific properties has so far largely been the result of trial and error: which is quite timeconsuming and low productive.

Things are about to change. Using combinatorial chemistry, CAS scientists demonstrated that bulk metallic glasses with new features can be obtained in a more efficient way. Read more on page 12.

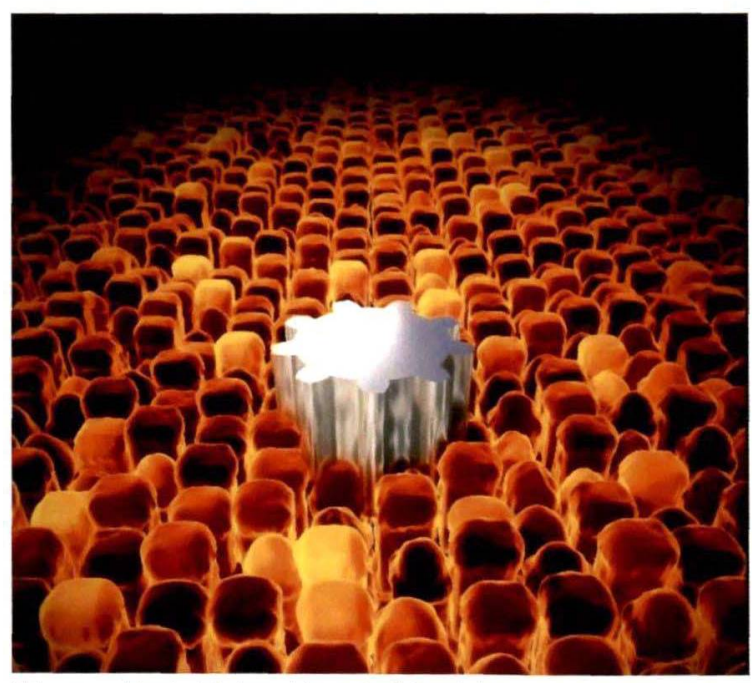

Using combinatorial chemistry, scientists established a high-throughput way to make a new class of high-temperature bulk metallic glasses by screening over a thousand of alloy candidates that vary slightly in chemical compositions on single silicon wafer. (Credit: IOP) 
th

\section{Denisovan from Qinghai-Tỉbet Plateau}

It seemed that Denisovans. an extinct species or subspecies of hominids living in Sia about the same time as Neanderthals. had left behind no material except for several fossil fragments from Denisova cave in the Ntai Mountains of Siberia. Yet they did leave phantomlike genetic imprints in modern Asian groups, suggesting their once prevalence on this continent. However: the scarcity of fossil record of them has made it difficult to further understand this mysterious species.

Thankfully, advanced method to analyzing ancient proteins has facilitated the identification of an otherwise unknown piece of material as a mandible from this mysterious hominid. Read more on page $1+$.

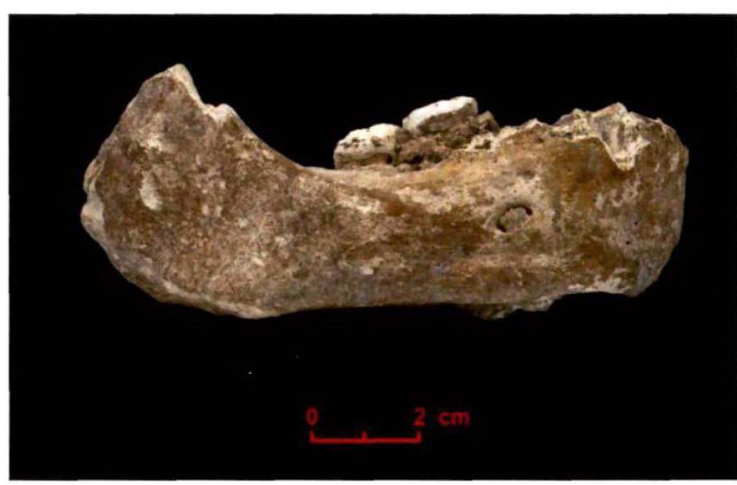

This once unknown fossil unearthed from Baishiya Karst cave, Xiahe Gansu, China, turns out to be a fragment of Denisovan mandible. (Credit: ITP)

$i^{\text {th }}$

\section{Disproval of Gravity-induced Quantum Decoherence by Micius in Space}

Quantum mechanics and general relativity are deemed two major "pillars" to support modern physics - they each are exceptionally successful in explaining phenomena in their own realms, namely the microscopic and the macroscopic worlds, respectively. However. any attempt to transcend the boundary to extend to the opposite has been frustrated with failure without any exception.

As efforts to bridge gravity and quantum fields. many rival models of quantum gravity have arisen, the "event formalism" is among them. Its prediction. however. failed to pass the test set by Micius. a satellite designed for quantum physics experiments flying at an altitude of about $500 \mathrm{~km}$ above the Earth. Read more on page 16 .

The Micius experiment tests the "event formalism" model by distributing entangled photon pairs across the space surrounding the Earth. For each pair, one photon is situated at the ground station and the other distributed to the satellite Micius. When the Earth rotates, the satellite will pass the ground station at different altitude, hence leaving the link between the twin photon of the pair cut through the space at different angles across different regions of the Earth's gravitationa fields. In this process, the pair undergoes fluctuation of the potential field and would decorrelate at some possibility if the "event formalism" was right - it proves to be not. (Credit: USIC)

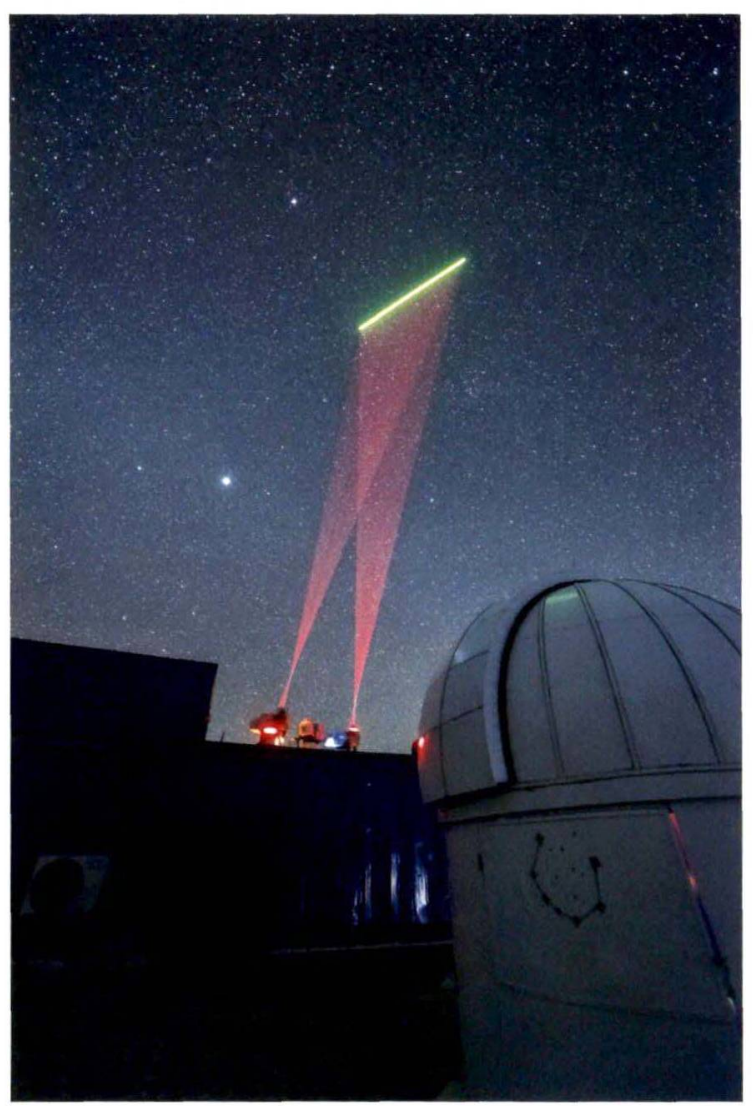




\section{The Architecture of African Swine Fever Virus}

The last year has witnessed numerous outbreaks of African swine fever virus (ASFV) worldwide, causing a huge economic loss. The virus specifically infects domestic pigs and wild boars. With no vaccine and treatments, nearly all infected animals ended in death and culling pigs have been so far the most effective way to contain its pandemics. To fight against this virus. there is an urgent demand to get to known it

Using single-particle cryo-electron microscopy. scientists at the CAS Institute of Biophysics (IBP) and their collaborators have elucidated a fine threedimension structure of ASFV and proposed a model for the virus assembly, which may provide clues for drug

\section{$10^{\text {th }}$}

\section{Observation of 3-D Quantum Hall Effect}

The discovery of the quantum Hall effect (QHE) in two-dimensional electronic systems in 1980 has rendered topology a central role in condensed matter physics. Its generalization to a three-dimensional system has intrigued physicists, and in 1987 Bertrand Halperin et al. demonstrated that it is theoretically possible. However, its experimental observation has proven to be challenging and no successful effort was reported until a joint team broke through.

Read how they managed to accomplish this on page 20 .

Three-dimensional quantum Hall effect observed in experiment (Credit: USTC)

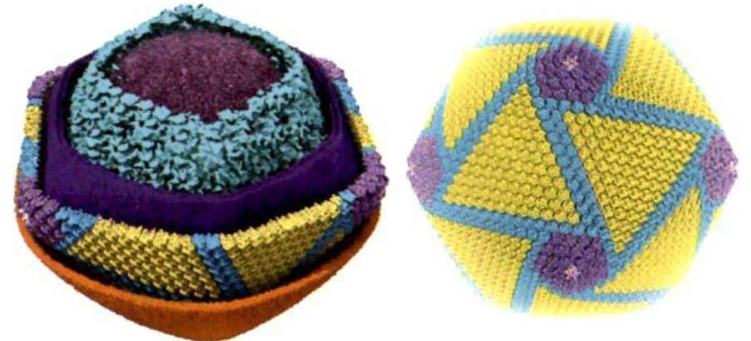

The five-layer structure of the African swine fever virus (ASFV) and how it looks at the capsid layer, a layer of protein shell. (Credit: CAS)

design and vaccine development. To have a good look of the architecture of this virus. please refer to page 18 .

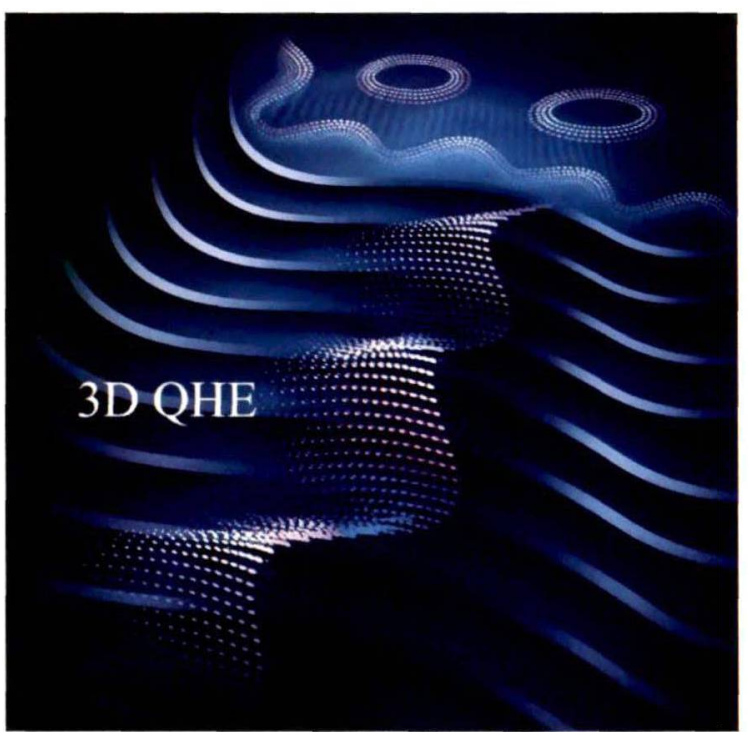




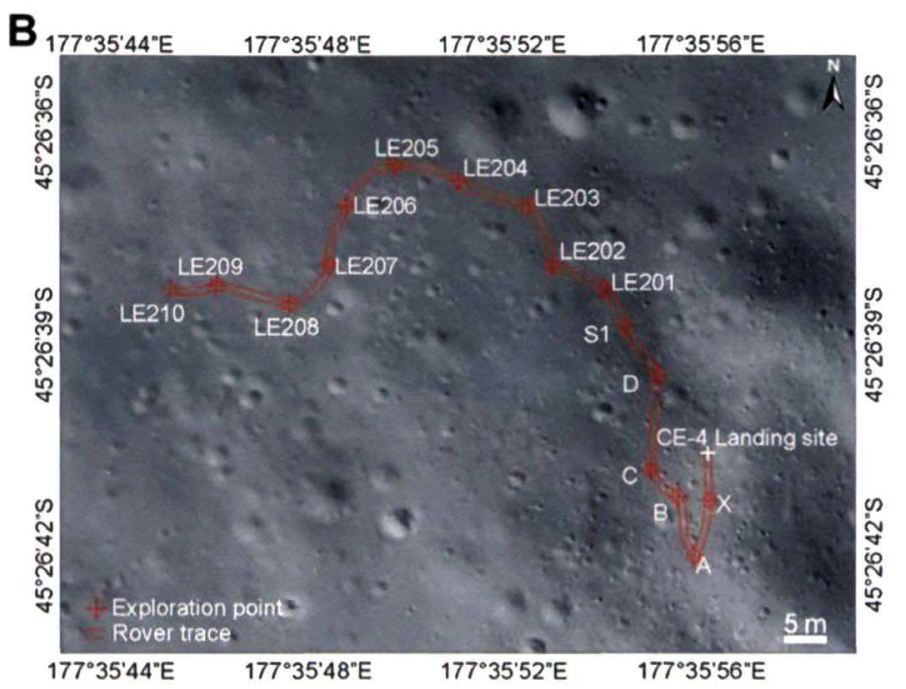

A series of exciting discoveries have been made concerning the chemical composition of the lunar mantle and the shallow subsurface structure of a lunar ejecta sequence, thanks to the data obtained during the in-situ observations performed by Yutu-2, the rover of Chang'e-4 mission under China's Lunar Exploration Program. The spacecraft touched down on the eastern floor of Von Kármán crater $\left(44.45^{\circ} \mathrm{S}, 176.3^{\circ} \mathrm{E}\right)$ in January 2019 , marking the first-ever soft landing by a manmade spacecraft on the far side of the Moon. The red lines, extending from point $A$ on the low right to point LE210 on the left, indicate the tracks of Yutu-2's left and right wheels during the first two lunar days. See page 4 and 8 for more. (Credit: CLEP/GRAS/NAOC) 